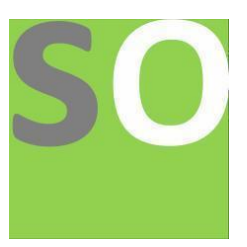

Article title: Recent research advances in disease models of Hutchinson-Gilford Progeria Syndrome Authors: Junzheng Yang[1]

Affiliations: Guangdong Nephrotic Drug Engineering Technology Research Center, The R\&D Center of Drug for Renal Diseases, Consun Pharmaceutical Group, Dongpeng Avenue, No.71, Guangzhou, Guangdong 510000, China.[1] Orcid ids: 0000-0001-9992-3179[1]

Contact e-mail: yangjunzheng606403@163.com

License information: This work has been published open access under Creative Commons Attribution License http://creativecommons.org/licenses/by/4.0/, which permits unrestricted use, distribution, and reproduction in any medium, provided the original work is properly cited. Conditions, terms of use and publishing policy can be found at https://www.scienceopen.com/.

Preprint statement: This article is a preprint and has not been peer-reviewed, under consideration and submitted to ScienceOpen Preprints for open peer review.

DOI: 10.14293/S2199-1006.1.SOR-.PPYLHY8.v1

Preprint first posted online: 30 January 2022

Keywords: HGPS, stem cell, iPSCs, disease models, aging. 


\title{
Recent research advances in disease models of Hutchinson-Gilford Progeria Syndrome
}

\author{
Junzheng Yang ${ }^{1 *}$ \\ ${ }^{1}$ Guangdong Nephrotic Drug Engineering Technology Research Center, The R\&D Center of Drug for \\ Renal Diseases, Consun Pharmaceutical Group, Dongpeng Avenue, No.71, Guangzhou, Guangdong \\ 510000, China. \\ *Correspondence to Junzheng Yang (yangjunzheng606403@163.com), Guangdong Nephrotic Drug \\ Engineering Technology Research Center, The R\&D Center of Drug for Renal Diseases, Consun \\ Pharmaceutic al Group, Guangzhou, Guangdong 510000, China.
}

\begin{abstract}
Aging can cause many diseases, and aging research may help to elucidate the pathogenesis and identify suitable therapies for attenuating aging or alleviating aging-related diseases such as Hutchinson-Gilford progeria syndrome (HGPS). HGPS is a sporadic genetic premature disorder caused by a de novo point mutation in the LMNA gene encoding the intermediate filament proteins lamins $\mathrm{A}$ and $\mathrm{C}$, typically resulting in a short lifespan (average age of 14.6 years). To date, there has been no effective therapy to cure it. In the past few decades, many scientists have focused on the development of disease models (such as iPSC, adult stem cell, and animal models) to uncover the underlying pathogenesis and find an effective therapy for HGPS. In this review, we summarize the recent advances in the disease models of HGPS, compare their advantages and disadvantages, and discuss the perspectives and challenges for these models, which may provide some clues for HGPS therapy.
\end{abstract}

Keywords: HGPS, stem cell, iPSCs, disease models, aging.

\section{Clinic characteristics of Hutchinson-Gilford progeria syndrome (HGPS)}

HGPS is a rare and fatal genetic disease with multiple clinical features and early premature phenotypes. This disease has a low incidence rate; typically, 1 case occurs in every 8 million live births. At birth, children with HGPS appear to be normal. However, within a year, they begin to develop symptoms related to the global premature phenotype, including hair loss, hypodermic fat loss, cardiovascular disease, and skeletal abnormalities; it is worth 
mentioning that there is no significant change in the central nervous system in patients with HGPS. HGPS is generally caused by the accumulation of progerin, which is a truncated form of lamin A precursor, a splicing variant of lamin A; progerin is produced by the incorrect activation of a cryptic splicing site in the LMNA gene. Upon full-length protein accumulation in HGPS and other laminin diseases, progerin affects nuclear tissue, chromatin dynamics, gene expression regulation, and epigenetic regulation. It has been previously demonstrated using primary dermal fibroblasts of patients with HGPS that an increase in progerin expression gradually damages cell proliferation and the cell cycle, indicating that the truncated form of prelamin $\mathrm{A}$ is one of the factors that accelerate the aging phenotype.

HGPS research is challenging because the incidence of HGPS is extremely low; hence, it is either difficult to obtain samples from patients with HGPS, or there generally exists a great ethical problem. Moreover, the skin of patients with HGPS is very fragile, and sample collection often does great harm to such patients. It is important to obtain reliable and sufficient HGPS samples for HGPS disease research; therefore, an HGPS disease model is necessary. In this review, we focus on the recent research progress in HGPS models, compare their advantages and disadvantages, and discuss the perspectives and challenges for these models, which may provide the foundation for HGPS research and therapy.

\section{Disease models of HGPS}

\subsection{Cell models of HGPS}

\subsubsection{Induced pluripotent stem cells (iPSCs) as a cell model for HGPS treatment and drug screening}

iPSCs are pluripotent cells that can differentiate into all types of body cells (neurons, cardiomyocytes (CMs), adipocytes, osteoblasts, and chondrocytes). In 2006, Takahashi K, et al. ${ }^{[1,2]}$ reported that iPSCs could be obtained from mouse embryonic or adult fibroblasts by introducing four transcription factors (Oct-3/4, Sox2, c-Myc, and Klf4). iPSCs have been attracting increasing attention from scientists; moreover, iPSCs have various applications in HGPS research. For example, Zhang J, et al. ${ }^{[3]}$ reprogrammed HGPS dermal fibroblasts into iPSCs and differentiated iPSCs into different types of cells, such as neural progenitors, endothelial cells, fibroblasts, vascular smooth muscle cells (VSMCs), and mesenchymal stem 
cells (MSCs). They found that progerin was highly expressed in PSC-derived MSCs, iPSC-derived VSMCs, and fibroblasts, and increased DNA damage; nuclear abnormalities were also found in these cell lines, especially in HGPS-VSMCs, in addition to the accumulation of numerous calponin-staining inclusion bodies. It is notable that HGPS-MSCs and HGPS-VSMCs were sensitive to stress and hypoxia in vitro and in vivo. Liu GH, et al. ${ }^{[4]}$ obtained iPSCs from fibroblasts of patients with HGPS, and found that HGPS- iPSCs (without progerin, lacking the nuclear envelope, and having epigenetic alterations normally associated with premature aging) could differentiate into SMCs with the appearance of premature senescence phenotypes associated with vascular aging, providing an iPSC-based model to study the pathogenesis of HGPS in vitro.

HGPS is a rare disease that is generally accompanied by many aging-associated syndromes such as alopecia, heart failure, lipodystrophy, osteoporosis, and atherosclerosis. To better understand its pathogenesis and identify a suitable therapy, a wide range of HGPS cell models have been generated using iPSC and gene editing technologies, such as iPSC-derived SMCs, neuron-like cells, and adipocytes, which act as a foundation for HGPS treatment and research, and help understand the aging process.

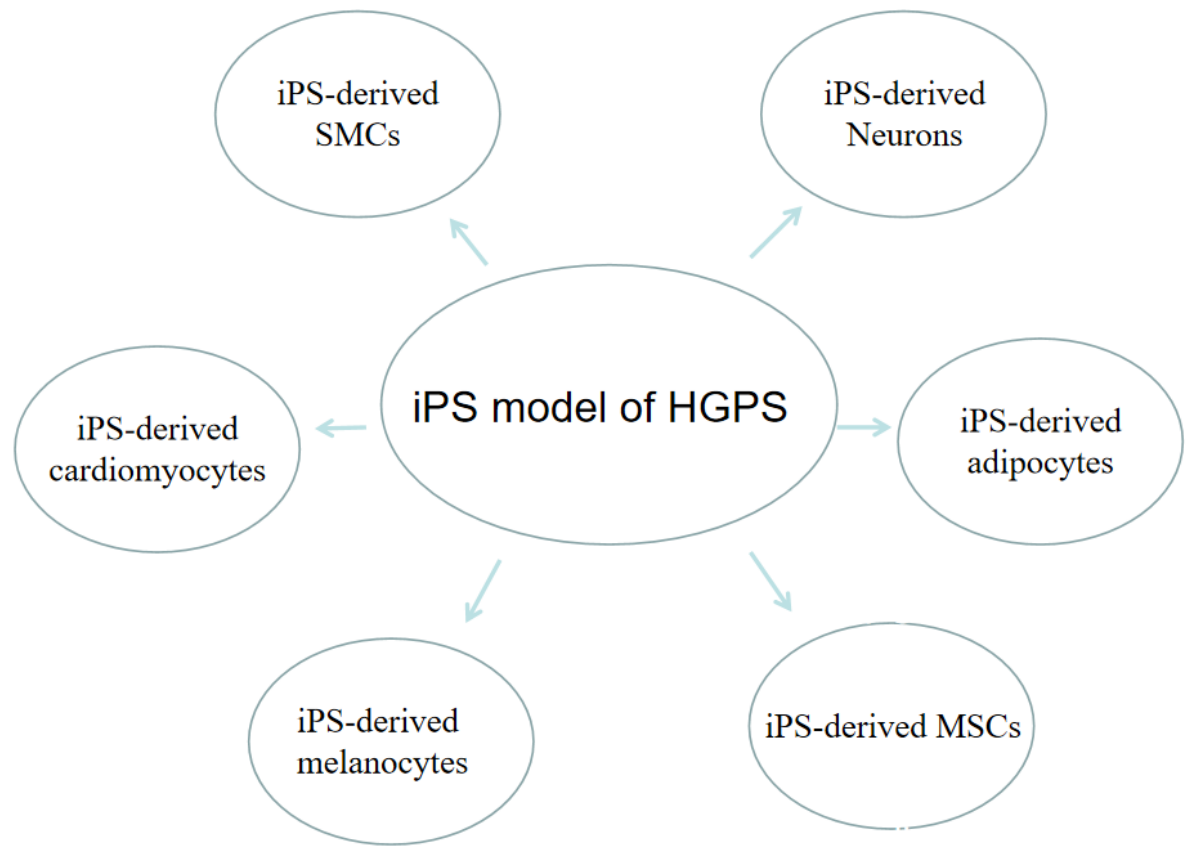

Figure 1. Summary of iPSC models of HGPS

\subsubsection{1 iPSC-derived SMCs as a cell model of HGPS}


It has been reported that VSMC senescence is closely related to advanced arteriosclerosis in normal populations, and progerin is known to accumulate mainly in arterial SMCs of patients with HGPS. Moreover, VSMC degeneration is one of the characteristics of HGPS-associated arteriosclerosis. As SMCs from patients with HGPS are very difficult to obtain, iPSC-derived SMCs may be a good tool for HGPS treatment research. To build an iPSC-based SMC model and study the pathogenesis of HGPS and physiological vascular aging in vitro, Zhang $\mathrm{J}$, et al. ${ }^{[3]}$ reprogrammed dermal fibroblasts of patients with HGPS into iPSCs, and differentiated the iPSCs into several types of body cells, such as neural progenitors, endothelial cells, fibroblasts, VSMCs, and MSCs; they found that progerin expression was higher in iPSC-derived MSCs, VSMCs, and fibroblasts, which showed premature senescence phenotypes. Liu GH, et al. ${ }^{[4]}$ reprogrammed fibroblasts of patients with HGPS into iPSCs, and then differentiated the HGPS-derived iPSCs into MSCs; the MSCs had the appearance of premature senescence phenotypes associated with vascular aging. In particular, iPSC-derived VSMCs accumulated more calponin-staining inclusion bodies than neural progenitors, endothelial cells, fibroblasts, and MSCs, and showed functional defects under stress and hypoxia. Zhang $\mathrm{H}$, et al. ${ }^{[5]}$ found that HGPS-derived SMCs exhibited a proliferative defect caused by caspase-independent cell death and prolonged mitosis. This demonstrated that PARP1 could mediate SMC loss in patients with HGPS, elucidating a molecular pathway underlying progressive SMC loss in progeria. To develop improved models and discover novel therapeutics to overcome the limitations of $2 \mathrm{D}$ cell culture and mouse models, Atchison L, et al. ${ }^{[6]}$ produced a functional 3D model of HGPS that replicated an arteriole-scale tissue-engineered blood vessel (TEBV) using iPSC-derived SMCs from a patient with HGPS. To pinpoint the effect of HGPS iSMCs, they used an endothelial layer consisting of human cord blood-derived endothelial progenitor cells (hCB-EPCs) from a healthy donor. The TEBVs fabricated from HGPS iSMCs and hCB-EPCs showed reduced vasoactivity, increased medial wall thickness, and increased calcification and apoptosis compared with TEBVs fabricated from normal iSMCs or primary MSCs. Additionally, treatment of HGPS TEBVs with the proposed therapeutic, everolimus, increased HGPS TEBV vasoactivity and iSMC differentiation in the TEBVs. These results demonstrate the ability of this iPSC-derived TEBV to reproduce key features of HGPS and respond to drugs. 
Bersini S, et al. ${ }^{[7]}$ generated induced vascular endothelial cells (iVECs) and iSMCs by direct reprogramming of healthy human fibroblasts of patients with HGPS to model vascular aging and HGPS in vitro; they found that iSMCs from patients with HGPS highly express BMP-4, the serum concentrations of which were high. Strikingly, blocking BMP-4 helped restore the vascular functionality; this may represent a potential future therapeutic strategy to limit cardiovascular dysfunction in HGPS.

\subsubsection{2 iPSC-derived neuron- like cells as a cell model of HGPS}

For several decades, many studies on HGPS focused on using peripheral cells, such as skin, blood, and bone marrow cells ${ }^{[8,9]}$, in vitro; these cells are not directly relevant for extrapolation to the behavior of other cell types, especially neural cell lineages. Hence, Nissan X, et al. ${ }^{[10]}$ differentiated iPSCs derived from cells of patient with HGPS into several neuron subtypes, and found that lamins $\mathrm{A} / \mathrm{C}$ were lacking in neurons of different subtypes. $\mathrm{A}$ microRNA, miR-9, negatively controls lamin A and progerin expression in neural cells, and miR-9 overexpression can alleviate nuclear blebbing in non-neural cells. Miller JD, et al. ${ }^{[11]}$ applied human iPSC-derived lineages to induce aging-related features in an animal model of Parkinson's disease (PD). They found that iPSC-derived fibroblasts and neurons could express progerin and multiple aging-related markers, especially dopamine-specific phenotypes (neuromelanin accumulation). PD iPSC-derived dopamine neurons exhibited pronounced dendrite degeneration, progressive loss of tyrosine hydroxylase (TH) expression, and enlarged mitochondria or Lewy-body-precursor inclusions. This type of hiPSC-based progerin-induced aging in disease model can be used to reveal late-onset age-related disease features.

\subsubsection{3 iPSC-derived CMs as a cell model of HGPS}

To date, there have been few studies on the effects of lamin A/C defects on human CMs. Crasto S, et al. ${ }^{[12]}$ generated an iPSC-derived cardiac model of laminopathy, which carried the K219T lamin A/C mutation. After in vitro and in vivo experiments, they found that these cells recapitulated the morphological features of dilated cardiomyopathy, specifically sarcomeric disorganization and increased size, and mutated lamin A/C perturbed $S C N 5 \mathrm{~A}$ gene expression by favoring the binding of PRC2 (Polycomb Repressive Complex 2) to its promoter, leading to decreased sodium current peak and slower conduction velocity. 


\subsubsection{PPS-derived adipocytes as a cell model of HGPS}

It has been reported that there are almost 13 diseases (known as laminopathies) resulting from $L M N A$ gene mutations ${ }^{[13]}$. HGPS is a laminopathy that generally produces very severe lipodystrophic symptoms with complete loss of subcutaneous fat ${ }^{[14]}$. Xiong Z-M, et al. ${ }^{[15]}$ reprogrammed HGPS fibroblasts into iPSCs, and differentiated iPSC-derived embryoid bodies or MSCs into adipocytes. As progerin inhibited the transcriptional activation of PPAR- $\gamma 2$ and $\mathrm{C} / \mathrm{EBP} \alpha$, adipocytes obtained from iPSC-derived embryoid bodies or MSCs exhibited a severe lipid storage defect at the late differentiation stage, but this had little effect on the early adipogenic regulators.

\subsubsection{5 iPSC-derived melanocytes as a cell model of HGPS}

To understand the relationship between pigmentation disorders and HGPS, Lo Cicero A, et al. ${ }^{[16]}$ derived two types of iPS cell lines from patients with HGPS and differentiated them into melanocytes. Compared with non-pathological cells, the HGPS melanocytes showed lower levels of melanin and a lower percentage of mature, fully pigmented melanosomes, thus demonstrating a direct role of progerin in the regulation of melanogenesis. These results reveal a new dysregulated pathway in HGPS and open up novel perspectives for the study of pigmentation phenotypes associated with normal and pathological aging.

\subsubsection{6 iPSC-derived MSCs as a cell model of HGPS}

To mimic HGPS, Wu Z, et al. ${ }^{[17]}$ generated human embryonic stem cells (ESCs) harboring a heterozygous LMNA p.G608G mutation and genetically enhanced HGPS-specific ESCs bearing a biallelic LMNA p.G608G mutation using gene editing technology. They found that HGPS-MSCs, but not ESCs or embryonic cells (ECs), exhibited typical aging-associated characteristics and distinct aging kinetics, providing a cell model for understanding the pathology of human aging and a platform for screening therapeutic strategies against aging-associated disorders. Cho S, et al. ${ }^{[18]}$ differentiated iPSCs derived from patients with HGPS into MSCs, and found that the stoichiometry of total A-type lamins (including progerin) versus B-type lamins was similar in progeria PSC-MSCs and normal MSCs. Transcription-regulating retinoids had an equal effect on both progeria iPSC-MSCs and normal A-type lamin MSCs, and the phosphorylation of progerin at multiple sites in iPSC-MSCs cultured on rigid plastic was lower than that of normal MSCs. Using HGPS 
iPSC-MSCs, Lo Cicero A, et al. ${ }^{[19]}$ screened seven compounds, including all-trans retinoic acid and 13-cis-retinoic acid, which could normalize osteogenic differentiation and decrease progerin expression, thus providing a type of cell model for high-throughput drug screening using HGPS iPSC-derived MSCs. For drug screening, Blondel S, et al. ${ }^{[20]}$ used MSCs derived from HGPS iPSCs to identify several pharmacological inhibitors of farnesylation that could alleviate pathological phenotypes associated with HGPS and demonstrated the unique potential applications of iPSC derivatives for drug screening. To test the effect of NRF2 on HGPS MSCs, Kubben N, et al. ${ }^{[21]}$ generated iPSCs from fibroblasts of patient with HGPS and found that reduced NRF2 activity decreased the expression of NRF2 antioxidant genes, increased oxidative stress, reduced the levels of lamin B1, LAP2, and HP1 $\gamma$ proteins, increased the frequency of senescence-associated $\beta$-galactosidase (SA- $\beta$-gal)-positive cells, and ultimately, reduced cell survival in HGPS-MSCs, which was sufficient to mimic HGPS defects.

\subsubsection{Adult stem cells as a cell model of HGPS}

In addition to iPSC models of HGPS, which are good tools for HGPS treatment or research, there are other models called adult stem cells (ASCs), which play a significant role in the human body, and are highly relevant to HGPS. For example, Pacheco LM, et al. ${ }^{[22]}$ isolated a well-defined, immature subpopulation of MSCs from the marrow and found that progerin protein plays an important role in vascular repair by regulating stem cell functions. MIAMI cells from a non-HGPS older donor could accumulate progerin in vivo, and a farnesyltransferase inhibitor could ameliorate these effects. This may be a feasible strategy for HGPS treatment through the downregulation of progerin using a farnesyltransferase inhibitor to disturb critical stem cell functions. Wenzel V, et al. ${ }^{[23]}$ reported an isolation method for naïve multipotent skin-derived precursor (SKP) cells from dermal fibroblast cultures from HGPS donors. These sphere cells could express nestin and multipotent markers (Oct-4, Sox2, Nanog, and TG30), and differentiate into SMCs (which could accumulate nuclear progerin with further passages) and fibroblasts. These results imply that progerin may cause aging through stem cell exhaustion. Scaffidi $\mathrm{P}$, et al. ${ }^{[24]}$ introduced progerin into immortalized human MSCs (hMSCs) and found that it interfered with their function by activating the Notch signaling pathway; the expression of progerin in hMSCs could change 
their molecular identity and differentiation potential, and several Notch effectors could cause endothelial dysfunction and aberrations of osteogenesis and adipogenesis in cell lines of patients with HGPS. The results demonstrated that adult stem cell dysfunction plays a significant role in accelerated aging in patients with HGPS.

\subsection{Animal models of HGPS}

In addition to cell models for understanding HGPS mechanisms or drug screening, animal models are another type of instrument for understanding HGPS mechanisms and for testing therapies.



Figure 2. Summary of animal models of HGPS

\subsubsection{Mouse models of HGPS}

HGPS mouse models are the main preclinical models for HGPS research, and many types of mouse models have been built to understand the mechanisms of HGPS. For example, Beyret E, et al. ${ }^{[25]}$ used transgenic mice expressing S. pyogenes Cas9 and exogenously delivered gRNAs and found that CRISPR-Cas9-mediated lamin A/progerin reduction by a single intravenous injection could enhance the health and extend the lifespan of HGPS mice.

Kreienkamp R, et al. ${ }^{[26]}$ found that a high-fat diet could more effectively reduce early lethality and ameliorate morbidity among progeria mice compared with a regular diet. 
Critically, feeding mice a high- fat diet delayed aging and nearly doubled the lifespan; this is the greatest lifespan extension recorded for progeria mice. To understand the contribution of the endothelium to cardiovascular HGPS pathology, Osmanagic-Myers S, et al. ${ }^{[27]}$ generated an endothelium-specific HGPS mouse model with selective endothelial progerin expression and found that progerin-induced impairment of mechanosignaling in endothelial cells contributes to excessive fibrosis and cardiovascular disease in patients with HGPS. Hamczyk MR, et al. ${ }^{[28]}$ generated a mouse model of progerin-induced atherosclerosis acceleration by crossing apolipoprotein E-deficient (Apoe-/-) mice with Lmna ${ }^{G 609 G / G 609 G}$ mice ubiquitously expressing progerin, crossed Apoe $e^{-/-} L m n a^{L C S / L C S}$ mice with LysMCre and SM22aCre mice to induce progerin expression specifically in macrophages or vascular smooth muscle cells (VSMCs) they provided evidences that VSMCs with restricting progerin expression result in accelerating atherosclerosis, triggering plaque vulnerability, and reducing lifespan. Gabriel Balmus, et al. ${ }^{[29]}$ demonstrated that Remodelin could significantly enhance the healthspan in a LmnaG609G HGPS mouse model through inhibition of the enzyme N-acetyltransferase 10 (NAT10). Osmanagic-Myers S, et al. ${ }^{[30]}$ generated an HGPS mouse model with specific endothelial progerin expression, the model showed clinical features of interstitial myocardial and perivascular fibrosis and left ventricular hypertrophy.

\subsubsection{Fish models of HGPS}

To investigate the role of lamin in fish species, Tonoyama Y, et al. ${ }^{[31]}$ generated zmpste24-deficient medaka using the TILLING method, and found that there were no growth defects or lifespan shortening during lamin precursor accumulation. The results showed that zmpste24-deficient medaka with an abnormal nuclear shape were hypersensitive to X-ray irradiation, and the telomere lengths were significantly shorter in the mutants than in the wild-type fish. These results suggest that the zmpste24-deficient medaka partially phenocopied HGPS, and that abnormal nuclear morphology and lifespan shortening are two independent events in vertebrates.

\subsubsection{Pig models of HGPS}

To better understand the mechanisms of HGPS after treatment, and observe the clinical effects, a large animal HGPS disease model has been built. Dorado B., et al. ${ }^{[32]}$ generated a Yucatan minipig HGPS model using the CRISPR-Cas9 gene editing technology. HGPS 
minipigs endogenously co-express progerin and normal lamin $\mathrm{A} / \mathrm{C}$, and exhibit severe growth retardation, lipodystrophy, skin and bone alterations, cardiovascular disease, and death around puberty. Remarkably, the HGPS minipigs recapitulate critical cardiovascular alterations observed in patients, such as left ventricular diastolic dysfunction, altered cardiac electrical activity, and loss of VSMCs.

\subsubsection{Monkey models of HGPS}

Genetically engineered animal models, specifically non-human primates, are a valuable tool for studying human diseases and developing preclinical therapeutic strategies for HGPS. Wang F, et al. ${ }^{[33]}$ generated a monkey model of HGPS by delivering a special base editor mRNA and guide RNA targeting the LMNA gene into monkey zygotes by microinjection. The results showed that five out of six newborn monkeys carried the LMNA gene mutation, and the HGPS monkeys that expressed progerin exhibited typical HGPS phenotypes (growth retardation, bone alterations, and vascular abnormalities). This monkey model may play a more significant role in HGPS research and clinic application than the current experimental models (mouse ${ }^{[30]}$, rabbit ${ }^{[34]}$, or pig ${ }^{[35]}$ model).

\section{Perspectives and challenges for HGPS disease models}

In this review, we summarized the recent research progress in HGPS disease models, including cell and animal models. The cell models include iPSC-derived, adult stem cell (ASC)-derived, and MSC-derived cell models; the animal models are divided into mouse, pig, rabbit, fish, and monkey models according to the species. Compared with animal models, cell models are easy to obtain and can be produced on a large scale to screen related drugs using high-throughput screening technology. However, they also have certain disadvantages. Cell models simulate the HGPS phenotype in vitro, but cannot simulate the complexity of HGPS in vivo; cell models are generally unstable and prone to mutation, and there are differences between cells.

Recently, researchers have paid great attention to HGPS because of its early onset and high mortality, and also because it is an excellent dise ase model for aging research. However, owing to the low incidence rate and the particularity of patient constitutions, tissues or cells from patients with HGPS are difficult to obtain, which greatly increases the difficulty of 
HGPS research. The rapid development of iPSC- and stem cell-related technology and animal model-building technology has largely contributed to in-depth studies on HGPS. However, there are still many problems: (1) the phenotype of HGPS cells can be simulated using iPSCs or stem cells to a large extent, but there are substantial differences at the gene level; (2) adult stem cells or MSCs can be induced to obtain specific cell types of HGPS, but the efficiency is very low; (3) the mouse model is the most widely used in HGPS research, but the current model poses a difficulty in simulating the recurrence of HGPS symptoms, which limits research on HGPS to a certain extent; (4) recently, a research team successfully developed a disease model of HGPS minipigs that exhibits good clinical characteristics, but this is still in the primary stage.

In summary, progress in science and technology and the in-depth study of HGPS will enable further understanding of this disease. Additionally, the development and improvement of HGPS disease models will provide a breakthrough in the treatment of HGPS and the underlying mechanisms of human aging.

\section{Author Disclosure Statement}

The authors declare they have no conflicting financial interests.

\section{Funding Information}

none

\section{References}

[1] Takahashi K, Yamanaka S. Induction of pluripotent stem cells from mouse embryonic and adult fibroblast cultures by defined factors [J] Cell, 2006, 126(4): 663-676.

[2] Takahashi K., Tanabe K., Ohnuki M., et al. Induction of pluripotent stem cells from adult human fibroblasts by defined factors [J]. Cell, 2007, 131(5):861-872.

[3] Zhang J, Lian Q, Zhu G, et al. A human iPSC model of Hutchinson Gilford Progeria reveals vascular smooth muscle and mesenchymal stem cell defects [J]. Cell Stem Cell, 2011, 8(1):31-45.

[4] Liu GH, Barkho BZ, Ruiz S, et al. Recapitulation of premature ageing with iPSCs from Hutchinson-Gilford progeria syndrome [J]. Nature, 2011, 14; 472(7342):221-225.

[5] Zhang H., Xiong Z. M., Cao K. Mechanisms controlling the smooth muscle cell death in progeria via down-regulation of poly (ADP-ribose) polymerase 1[J]. Proc. Natl. Acad. Sci. U.S.A., 111, E2261-E2270. 
[6] Leigh Atchison, Haoyue Zhang, Kan Cao, et al. A Tissue Engineered Blood Vessel Model of Hutchinson-Gilford Progeria Syndrome Using Human iPSC-derived Smooth Muscle Cells[J]. Sci Rep. 2017, 7: 8168 .

[7] Bersini S, Schulte R, Huang L, et al. Direct reprogramming of human smooth muscle and vascular endothelial cells reveals defects associated with aging and Hutchinson-Gilford progeria syndrome[J]. 2020, 8. doi: $10.7554 /$ eLife. 54383 .

[8] T. Misteli, P. Scaffidi. Genome instability in progeria: when repair gets old[J]. Nat. Med., 2005, 11: 718-719.

[9] M. Paradisi, D. McClintock, R.L. Boguslavsky, C. et al. Dermal fibroblasts in Hutchinson-Gilford progeria syndrome with the lamin A G608G mutation have dysmorphic nuclei and are hypersensitive to heat stress[J]. BMC Cell Biol., 2005, 6: 27.

[10] Nissan X, Blondel S, Navarro C, et al. Unique preservation of neural cells in Hutchinson- Gilford progeria syndrome is due to the expression of the neural-specific miR-9 microRNA[J]. Cell Rep., 2012, 2(1):1-9.

[11] Miller JD, Ganat YM, Kishinevsky S, et al. Human iPSC-based modeling of late-onset disease via progerin-induced aging [J]. Cell Stem Cell, 2013, 13(6): 691-705.

[12] Silvia Crasto, Nicolò Salvarani, Michele Miragoli, et al. Lamin A/C Mutations Epigenetically Dysregulate Scn5a Gene Expression, Perturbing Action Potential Properties in iPSC-derived Cardiomyocytes [J]. Circulation Research, 2018, 121: A11.

[13] Capell BC, Collins FS. Human laminopathies: nuclei gone genetically awry [J]. Nat Rev Genet, 2006, 7: 940-952.

[14] Hennekam RC. Hutchinson-Gilford progeria syndrome: review of the phenotype [J]. Am J Med Genet A, 2006, 140(23): 2603-2624.

[15] Zheng-Mei Xiong, Christina LaDana, Di Wu, et al. An inhibitory role of progerin in the gene induction network of adipocyte differentiation from iPS cells [J]. Aging (Albany NY), 2013, 5(4): 288-303.

[16] Lo Cicero A, Saidani M, Allouche J, et al. Pathological modelling of pigmentation disorders associated with Hutchinson-Gilford Progeria Syndrome (HGPS) revealed an impaired melanogenesis pathway in iPS-derived melanocytes [J]. Sci Rep., 2018, 8(1): 9112. 
[17] Wu Z, Zhang W, Song M, et al. Differential stem cell aging kinetics in Hutchinson-Gilford progeria syndrome and Werner syndrome [J]. Protein Cell, 2018, 9(4): 333-350.

[18] Cho S, Abbas A, Irianto $\mathrm{J}$, et al. Progerin phosphorylation in interphase is lower and less mechanosensitive than lamin-A,C in iPS-derived mesenchymal stem cells [J]. Nucleus, 2018, 9(1): 230-245.

[19] Lo Cicero A, Jaskowiak AL, Egesipe AL, et al. A High Throughput Phenotypic Screening reveals compounds that counteract premature osteogenic differentiation of HGPS iPS-derived mesenchymal stem cells [J]. Sci Rep., 2016, 6: 34798.

[20] Blondel S, Egesipe AL, Picardi P, et al. Drug screening on Hutchinson Gilford progeria pluripotent stem cells reveals aminopyrimidines as new modulators of farnesylation [J]. Cell Death Dis., 2016, 7:e2105.

[21] Kubben N, Zhang W, Wang L, et al. Repression of the Antioxidant NRF2 Pathway in Premature Aging [J]. Cell, 2016, 165(6):1361-1374.

[22] Laurin Marie Pacheco, Lourdes Adriana Gomez, Janice Dias, et al. Progerin expression disrupts critical adult stem cell functions involved in tissue repair [J]. Aging (Albany NY), 2014, 6(12): $1049-1063$.

[23] Wenzel V, Roedl D, Gabriel D, et al. Naïve adult stem cells from patients with Hutchinson-Gilford progeria syndrome express low levels of progerin in vivo [J]. Biol Open, 2012, 1(6): 516-526.

[24] Scaffidi P, Misteli T. Lamin A-dependent misregulation of adult stem cells associated with accelerated ageing [J]. Nat Cell Biol., 2008, 10(4):452-459.

[25] Ergin Beyret, Hsin-Kai Liao, Mako Yamamoto, et al. Single-dose CRISPR-Cas9 therapy extends lifespan of mice with Hutchinson-Gilford progeria syndrome [J]. Nature Medicine, 2019, 25: 419-422.

[26] Ray Kreienkamp, Cyrielle Billon, Gonzalo Bedia-Diaz, et al. Doubled lifespan and patient-like pathologies in progeria mice fed high-fat diet [J]. Aging Cell, 2019, 18(1): e12852.

[27] Selma Osmanagic-Myers, Attila Kiss, Christina Manakanatas, et al. Endothelial progerin expression causes cardiovascular pathology through an impaired mechanoresponse [J]. J Clin Invest, 2019, 129(2): $531-545$.

[28] Hamczyk MR, Villa-Bellosta R, Gonzalo P, et al. Vascular Smooth Muscle-Specific Progerin Expression Accelerates Atherosclerosis and Death in a Mouse Model of Hutchinson-Gilford Progeria Syndrome [J]. Circulation, 2018, 138(3): 266-282. 
[29] Gabriel Balmus, Delphine Larrieu, Ana C. Barros, et al. Targeting of NAT10 enhances healthspan in a mouse model of human accelerated aging syndrome [J]. Nat Commun., 2018, 9: 1700.

[30] Osmanagic-Myers S, Kiss A, Manakanatas C, et al. Endothelial progerin expression causes cardiovascular pathology through an impaired mechanoresponse [J]. J Clin Invest., 2019, 129 (2):531-545. [31] Tonoyama Y, Shinya M, Toyoda A, et al. Abnormal nuclear morphology is independent of longevity in a zmpste24-deficient fish model of Hutchinson-Gilford progeria syndrome [J]. Comp Biochem Physiol C Toxic ol Pharmacol., 2018, 209: 54-62.

[32] Beatriz Dorado, Gro Grunnet Pløen, Ana Barettino, et al. Generation and characterization of a novel knockin minipig model of Hutchinson-Gilford progeria syndrome [J]. Cell Discov., 2019, 5: 16.

[33] Wang F, Zhang W, Yang Q, et al. Generation of a Hutchinson-Gilford progeria syndrome monkey model by base editing [J]. Protein cell, 2020, 11(11):809-824.

[34] Osorio FG, Navarro CL, Cadinanos J, et al. Splicing-directed therapy in a new mouse model of human accelerated aging [J]. Sci Transl Med., 2011, 3:106ra107.

[35] Liu Z, Chen M, Chen S, et al. Highly efficient RNA-guided base editing in rabbit [J]. Nat Commun., 2018, 9:2717. 